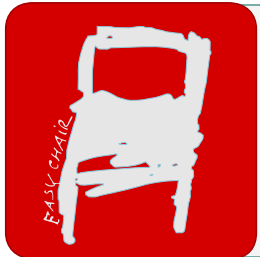

EPiC Series in Health Sciences

Volume 3, 2019, Pages 166-168

CAOS 2019. The 19th Annual Meeting of the International Society for Computer Assisted Orthopaedic Surgery

\title{
The Impact of Workflow on Cutting Time for Partial Knee Arthroplasty
}

\author{
Emily L. Hampp $\mathrm{PhD}^{1}$, Laura Y. Scholl $\mathrm{MSc}^{1}$, Md Haque $\mathrm{PhD}^{1}$, Chunyan $\mathrm{Wu} \mathrm{PhD}^{1}$, Geoffrey \\ Westrich $\mathrm{MD}^{2}$ and Michael A. Mont $\mathrm{MD}^{3}$ \\ ${ }^{1}$ Stryker, Mahwah, NJ, USA \\ ${ }^{2}$ Hospital for Special Surgery, New York, NY, USA \\ ${ }^{3}$ Lenox Hill, New York, NY, USA \\ emily.hamppestryker.com, laura.shollestryker.com, \\ md.haque@stryker.com, chunyan.Wu@stryker.com, WestrichG@HSS.EDU, \\ mmontanorthwell.edu
}

\begin{abstract}
To date, there are no studies aimed at characterizing the active cutting time for a robotic-assisted PKA procedure and the impact of workflow. This study quantified the active cutting time for three medial PKA workflows using the same robotic-assisted system.

Three surgeons each prepared six cadaveric knees for PKA with robotic-assisted technology using one of three workflows: A) burr-only, using a legacy cutting system and burr design; B) burr-only, using a new cutting system and burr design; or C) planar, using a new cutting system, burr design and saw. For a burr-only workflow, the femur and tibia were prepared with a burr. For a planar workflow, the femur and tibia were prepared with a burr and saw. The total mean trigger time to complete all femoral or tibial bony resections was measured and statistically compared between workflows using ANOVA and Tukey Pairwise Comparison.

There was statistically significant less time required to prepare the femur and tibia in $\mathrm{B}$ and $\mathrm{C}$, compared to A $(\mathrm{p} \leq 0.05)$. Less time was required in $\mathrm{C}$ than $\mathrm{B}$, but this was not statistically significant ( $>0.05$ ). Workflow A took an average of $429 \pm 104$ seconds (range, 314 to 529 seconds), B took an average of $302 \pm 40$ seconds (range, 244 to 363 seconds), and $C$ took an average of $236 \pm 50$ seconds (range, 196 to 332 seconds).

The new burr design, when used with both burr-only and planar workflows, has the potential to be more efficient ( 30 and $45 \%$ faster, respectively) compared to the legacy burr using the burr-only workflow, for a medial PKA. Bone resection time should be investigated in a clinical setting.
\end{abstract}




\section{Introduction}

Partial knee arthroplasty (PKA), also termed unicompartmental knee arthroplasty (UKA) when associated with a single compartment, has been performed for isolated single compartment knee osteoarthritis (OA) since the 1970s.[1] PKA can be carried out in the medial, lateral or patellofemoral (PF) compartments. When compared to total knee arthroplasty (TKA), studies have shown that medial PKA patients have experienced greater retention of normal knee kinematics and accelerated recovery, while suffering less blood loss and reduced post-operative morbidity.[2-5]

Despite the evidence demonstrating the benefits of PKA, there remains a desire to decrease operative times without sacrificing the ability to achieve high accuracy. To date, there are no studies aimed at characterizing the active cutting time for a robotic-assisted PKA procedure and the impact of workflow. This study quantified the active cutting time for three robotic-assisted medial PKA workflows.

\section{Materials and Methods}

Three surgeons each prepared six cadaveric knees for PKA (for a total of 18 knees) with roboticassisted technology using one of three workflows. Efforts were made to limit operator variability by selecting three surgeons who had prior exposure to robotic-assisted PKA surgery. Each surgeon was assigned the configurations shown in Table A for each of the three sets of cadavers.

Table A: Specimen configurations

\begin{tabular}{|c|c|c|}
\hline Cadaver specimen & Left & Right \\
\hline 1 & Workflow A & Workflow C \\
\hline 2 & Workflow B & Workflow A \\
\hline 3 & Workflow C & Workflow B \\
\hline
\end{tabular}

The assignments were intended to distribute procedures between specimens to eliminate any potential baseline variability from a single specimen, by splitting procedures across the specimens to have no two identical preparations between the left and right knees of the same specimen. There was an equal number of left and right cases for Workflow A, B and C. Further, attempt was made to evenly distribute $\mathrm{OA}$ between surgeon and surgical application based on grading of the pre-operative X-rays.

The three workflows investigated were: Workflow A) burr-only, using a legacy cutting system and burr design; Workflow B) burr-only, using a new cutting system and burr design; and Workflow C) planar, using a new cutting system, burr design and saw. For a burr-only workflow, the femur and tibia are prepared with a burr. For a planar workflow, the femur and tibia are prepared with a burr and saw.

The total mean trigger time, or the active cutting time, to complete all femoral or tibial bony resections was measured and statistically compared between workflows using ANOVA and Tukey Pairwise Comparison.

\section{Results}

There was statistically significant less time required to prepare the femur and tibia in B and C, compared to $A(p \leq 0.05)$. Less time was required in $C$ than $B$, but this was not statistically significant ( $>>0.05$ ). Workflow A took an average of $429 \pm 104$ seconds (range, 314 to 529 seconds), B took an average of $302 \pm 40$ seconds (range, 244 to 363 seconds), and C took an average of $236 \pm 50$ seconds (range, 196 to 332 seconds). 


\section{Discussion and Conclusion}

Past studies have demonstrated that robotic-assisted technology has enabled the surgeon to accurately and consistently place the femoral and tibial PKA components in accordance with preoperative plans and to effectively restore soft tissue balancing.[6-7] This technology is associated with a short learning curve to achieve time neutral surgery compared to manual surgery, without influencing the ability to achieve high accuracy.[8] However, the desire still remains to decrease operative times.

In this study, the new burr design, when used with both burr-only and planar workflows, has the potential to be more efficient (30 and $45 \%$ faster, respectively) compared to the legacy burr using the burr-only workflow, for a medial PKA. For further confirmation, bone resection time should be investigated in a clinical setting.

\section{References}

[1] Ollivier M, Abdel M, Parratte S, and Argenson J-N. Lateral unicondylar knee arthroplasty (UKA): Contemporary indications, surgical technique, and results. Int Orthop. 2014;38(2):449-455.

[2] Price AJ, Rees JL, Beard DJ, Gill RH, Dodd CA, Murray DM. Sagittal plane kinematics of a mobile-bearing unicompartmental knee arthroplasty at 10 years: a comparative in vivo fluoroscopic analysis. The Journal of arthroplasty. 2004;19(5):590-7.

[3] Schwab PE, Lavand'homme P, Yombi JC, Thienpont E. Lower blood loss after unicompartmental than total knee arthroplasty. Knee surgery, sports traumatology, arthroscopy: official journal of the ESSKA. 2015;23(12):3494-500.

[4] Brown NM, Sheth NP, Davis K, Berend ME, Lombardi AV, Berend KR, et al. Total knee arthroplasty has higher postoperative morbidity than unicompartmental knee arthroplasty: a multicenter analysis. The Journal of arthroplasty. 2012;27(8 Suppl):86-90.

[5] Larsen K, Sorensen OG, Hansen TB, Thomsen PB, Soballe K. Accelerated perioperative care and rehabilitation intervention for hip and knee replacement is effective: a randomized clinical trial involving 87 patients with 3 months of follow-up. Acta orthopaedica. 2008;79(2):149-59.

[6]. Bell SW; Anthony I; Jones B; MacLean A; Rowe P; Blyth M. Improved accuracy of component positioning with robotic-assisted unicompartmental knee arthroplasty: data from a prospective, randomized controlled study. J Bone and Joint Surg. 2016;98: 627-35.

[7]. Plate JF, Mofidi A, Mannava S, Smith BP, et al. Achieving accurate ligament balancing using robotic-assisted unicompartmental knee arthroplasty. Advances in Orthopedics. 2013;837167.

[8]. Kayani D, Konan S, Pietrzak JRT, Huq SS, Tahmassebi J, Haddad FS. The learning curve associated with robotic-arm assisted unicompartmental knee arthroplasty. Bone Joint J 100-B. $2018 ; 1033-42$ 